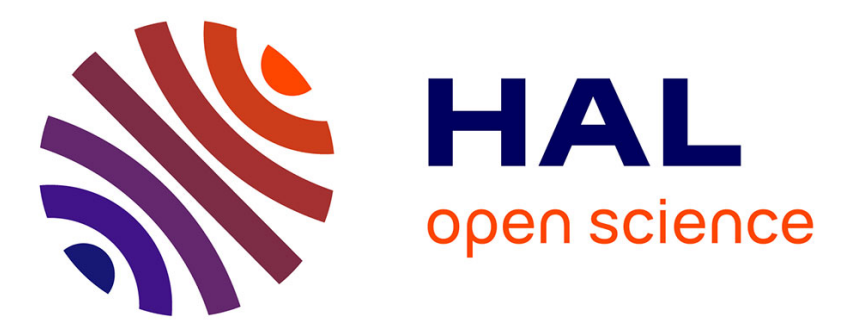

\title{
Increased intra-cortical porosity reduces bone stiffness and strength in pediatric patients with osteogenesis imperfecta
}

V. Vardakastani, Dominique Saletti, W. Skalli, P. Marry, Jean-Marc Allain, C. Adam

\section{To cite this version:}

V. Vardakastani, Dominique Saletti, W. Skalli, P. Marry, Jean-Marc Allain, et al.. Increased intracortical porosity reduces bone stiffness and strength in pediatric patients with osteogenesis imperfecta. BONE, 2014, 69, pp.61-67. 10.1016/j.bone.2014.09.003 . hal-03198116

\section{HAL Id: hal-03198116 https://hal.science/hal-03198116}

Submitted on 14 Apr 2021

HAL is a multi-disciplinary open access archive for the deposit and dissemination of scientific research documents, whether they are published or not. The documents may come from teaching and research institutions in France or abroad, or from public or private research centers.
L'archive ouverte pluridisciplinaire HAL, est destinée au dépôt et à la diffusion de documents scientifiques de niveau recherche, publiés ou non, émanant des établissements d'enseignement et de recherche français ou étrangers, des laboratoires publics ou privés. 


\title{
Rapid Communication \\ Increased intra-cortical porosity reduces bone stiffness and strength in pediatric patients with osteogenesis imperfecta
}

\author{
V. Vardakastani a , D. Saletti a, W. Skalli a , P. Marry ${ }^{\text {b }}$, J.M. Allain ${ }^{\text {c }}$, C. Adam ${ }^{\text {a,d,* }}$ \\ a Institut de Biomécanique Humaine Georges Charpak, Arts et Metiers ParisTech (ENSAM), 151 Boulevard de l'Hôpital, 75013 Paris, France \\ b Service de Chirurgie Orthopédique et Réparatrice de l'enfant, Hôpital Armand Trousseau, 26, avenue du Docteur Arnold Netter, 75571 Paris Cedex 12 , France \\ c Laboratoire de Mécanique des Solides, CNRS UMR7649, Ecole Polytechnique, 91128 Palaiseau Cedex, France \\ d School of Chemistry, Physics and Mechanical Engineering, Queensland University of Technology, GPO Box 2434, 2 George St, Brisbane, Australia
}

Keywords:

Osteogenesis imperfecta

Bone mechanics

Bone microstructure

Intra-cortical porosity

Collagen alignment

\begin{abstract}
A B S T R A C T
Osteogenesis imperfecta (OI) is a heritable disease occurring in one out of every 20,000 births. Although it is known that Type I collagen mutation in OI leads to increased bone fragility, the mechanism of this increased susceptibility to fracture is not clear. The aim of this study was to assess the microstructure of cortical bone fragments from patients with osteogenesis imperfecta (OI) using polarized light microscopy, and to correlate microstructural observations with the results of previously performed mechanical compression tests on bone from the same source. Specimens of cortical bone were harvested from the lower limbs of three (3) OI patients at the time of surgery, and were divided into two groups. Group 1 had been subjected to previous micromechanical compression testing, while Group 2 had not been subjected to any prior testing. Polarized light microscopy revealed disorganized bone collagen architecture as has been previously observed, as well as a large increase in the areal porosity of the bone compared to typical values for healthy cortical bone, with large (several hundred micron sized), asymmetrical pores. Importantly, the areal porosity of the OI bone samples in Group 1 appears to correlate strongly with their previously measured apparent Young's modulus and compressive strength. Taken together with prior nanoindentation studies on OI bone tissue, the results of this study suggest that increased intra-cortical porosity is responsible for the reduction in macroscopic mechanical properties of OI cortical bone, and therefore that in vivo imaging modalities with resolutions of $\sim 100 \mu \mathrm{m}$ or less could potentially be used to non-invasively assess bone strength in OI patients. Although the number of subjects in this study is small, these results highlight the importance of further studies in OI bone by groups with access to human OI tissue in order to clarify the relationship between increased porosity and reduced macroscopic mechanical integrity.
\end{abstract}

\section{Introduction}

Osteogenesis imperfecta (OI) is a heritable disease that appears in one in 20,000 births. The disease is caused by a mutation of Type I collagen, and seven subtypes of OI have been identified [1]. The symptoms and severity of the disease vary between patients, but the main shared characteristic of $\mathrm{OI}$ is bone fragility, leading to a high risk of fracture. As human specimens of OI bone are difficult to obtain, limited information is available in existing literature regarding the mechanism of this decrease in bone mechanical integrity. A number of previous studies have performed nanoindentation testing on human OI bone specimens [2-6] and each of these studies has concluded that both the elastic modulus and hardness of the bone tissue itself are only marginally different to typical values for healthy cortical bone, despite the fact that it is

* Corresponding author at: Institut de Biomécanique Humaine Georges Charpak, Arts et Metiers ParisTech (ENSAM), 151 Boulevard de l'Hôpital, 75013 Paris, France.

E-mail address: clayton.adam@ensam.eu (C. Adam). known that OI significantly degrades the macroscopic mechanical behavior of the bone [5].

The fact that OI bone exhibits essentially normal stiffness and strength at the nanoscale suggests that the cause of the degraded macroscopic mechanical properties occurs at scales above that of the collagen/apatite nanostructure. That is to say, micro-morphological factors may contribute to the abnormal macroscopic behavior of OI cortical bone. For instance, there are suggestions in previous studies that abnormal collagen orientation and lamellar architecture affect bone mechanical integrity in OI, and decreased lamellar thickness and osteonal size have been reported in OI [7-10]. Moreover, the formation of micro-cracks and accumulation of micro-damage can also degrade mechanical behavior. Previous mouse studies $[8,11]$ indicate that OI bone shows a greater propensity to accumulate micro-damage and to form linear micro-cracks than normal bone. Bulk degradation of bone properties due to micro-cracks would not necessarily be detected by nanoindentation, whereas in a macroscopic mechanical test they would behave as material defects. Thirdly, there is the observation that cortical 
bone in OI becomes 'trabecularized' [12], so that although the matrix itself may not be mechanically compromised, the increased porosity could degrade macroscopic mechanical properties.

Given the apparent importance of microstructural alterations in OI, the aim of this study was to examine the microstructure of OI cortical bone in a series of biopsy specimens from human subjects using polarized light microscopy. Collagen fiber organization was qualitatively assessed, and areal intra-cortical porosity was calculated. Porosity was then compared to micro-mechanical stiffness and strength measured during previously performed compression tests on a subset of the specimens.

\section{Materials and methods}

\section{Specimen acquisition}

The human OI cortical bone specimens used in this study were harvested during surgical rodding procedures for the fixation of femoral and tibial fractures undertaken at Hospital Armand Trousseau in Paris, France. The study protocol was approved by the Hospital ethical committee and written parental consent was obtained for each patient. After harvest, the biopsy specimens were wrapped in saline soaked gauze and frozen at $-18{ }^{\circ} \mathrm{C}$ prior to transport to the laboratory.

\section{Group assignment}

A subset of the specimens described in the present study had been subjected to mechanical compression testing after harvest as part of a prior (unpublished) study. These specimens were assigned to Group 1. Group 2 comprised the remaining specimens which had not been previously compression tested, thereby allowing assessment of microstructure in untested specimens (since mechanical compression to failure could potentially affect microstructure, even though the final compressive strain was generally only a few percent). Since the protocol for the mechanical compression tests mentioned above has not been previously published, it is described below. The reader is referred to [13] for further detail of the compression tests from the unpublished thesis. After mechanical compression testing of the specimens in Group 1, all specimens from both groups were prepared for polarized light microscopy as described in the section 'Resin embedding' below.

\section{Mechanical compression testing of Group 1 specimens}

Unconfined compression tests were performed on the specimens assigned to Group 1 in the present study using a uniaxial RAITH ${ }^{\circledR}$ testing device [14]. Since the surgically harvested biopsy fragments were of irregular size and shape, prior to testing each specimen was thawed and cut under constant irrigation into a parallelepiped shape using a diamond saw (Isomet Low Speed Saw, Buehler, USA). Due to the variation in size of the biopsy fragments, the resulting parallelepipeds also varied in size. Dimensions of the prepared specimens varied from 1.3 to $4.3 \mathrm{~mm}$ in width and thickness, and from 5.0 to $6.9 \mathrm{~mm}$ in length. When biopsy fragments were too thin to be securely held for cutting with the diamond saw, specimen preparation was achieved using manual polishing with successively finer polishing disks until a flat surface had been achieved. For each specimen, the cutting direction was chosen such that the faces of the parallelepiped test specimen were approximately parallel and perpendicular to the fabric direction of the host bone tissue respectively, as ascertained by visual inspection of the microstructure of the harvested fragments prior to cutting. Each specimen was then oriented in the uniaxial testing apparatus such that the applied loading was approximately aligned with the fiber direction (primary loading axis) of the bone in vivo. Prior to each compression test, black ink dots were applied to the side of the specimen to aid in subsequent image analysis using digital image correlation for strain determination, then the sample was preloaded with three cycles of compression to a maximum force of $20 \mathrm{~N}$. Preloading helps to remove any artifacts due to minor asperities on the specimen surfaces. Compression tests were then performed at a strain rate of $0.001 \mathrm{~s}^{-1}$ until failure. During testing, force was measured using a $1 \mathrm{kN}$ load cell, and specimen deformation was imaged using a digital camera (Canon EOS, Canon Inc., Japan). After testing, successive images were processed to derive compressive axial strain using custom-written digital image correlation post-processing software [13]. Using the apparent stress $v s$. strain data thus derived, the apparent level Young's modulus was identified by least-squares fitting of the middle third of the linear region of the apparent stress $v s$. strain curve. The ultimate stress was identified as the maximal nominal stress obtained during each test. Note that when reporting mechanical test results, we use the terms 'apparent' elastic modulus and 'apparent' ultimate strength, to refer to the fact that the scale being tested is above that of the tissue level (as would be interrogated by nanoindentation), therefore the apparent properties are a function of both tissue material properties and microstructure.

\section{Resin embedding}

Prior to specimen preparation for microscopy, all specimens in both tested and untested groups were fixed in 10\% phosphate buffered formalin (Electron Microscopy Sciences, USA) at room temperature for $24 \mathrm{~h}$. Specimens were placed under vacuum at -300 mbar in order to assist formalin penetration. During fixation, the volume ratio of fixative to tissue was kept at or above 10:1. After fixation, all specimens were rinsed under running tap water for $1 \mathrm{~h}$. Specimens were then dehydrated in increasing concentrations of acetone (50\%; 75\%; $100 \%$ ), at $24 \mathrm{~h}$ per step based on the specimen size [15]. Specimens were then embedded in epoxy resin (Spurr Low Viscosity Embedding Media, Electron Microscopy Sciences, USA) using the corrected formulation of Ellis [16]. Resin impregnated specimens were mounted by placing them inside small rings of plastic which had been pre-glued onto standard $25 \times 75 \mathrm{~mm}$ microscopic slides, and then filling the space around the specimen with resin. After placement, specimens were degassed under vacuum for $15 \mathrm{~min}$ at $-300 \mathrm{mbar}$, and were then polymerized overnight at $60{ }^{\circ} \mathrm{C}$.

\section{Computer numerically controlled milling}

After embedding in epoxy resin, a custom built computer numerically controlled (CNC) milling system based on a Proxxon MF70 milling machine (Proxxon Ltd, Germany) was used to mill the mounted OI bone specimens down to the desired thickness for transmitted polarized light microscopy using natural birefringence (200-300 $\mu \mathrm{m})$. A $3 \mathrm{~mm}$ diameter, 4-flute milling bit was used in all milling processes in order to achieve surface finishes in the order of 5-10 $\mu \mathrm{m}$, which is less than the depth of field of the microscope at the magnifications used for imaging bone microstructure. This system allows preparation of large sections of mineralized bone at thicknesses of several hundred microns, suitable for polarized light imaging of interference colors in unstained specimens.

\section{Polarized light microscopy and image analysis}

Since structured arrays of collagen molecules are birefringent, collagen fibers in bone generate interference colors between crossed polarizers, with the color (or intensity in a single wavelength illumination system) containing information about specimen retardation, which can in turn be related to collagen orientation. Although we note that previous studies have quantitatively linked polarization colors to collagen orientation in bone $[17,18]$, in this study polarized light imaging was used qualitatively to provide an indication of the degree of organization of collagen in the bone, and quantitatively in the sense of providing unstained contrast between bone and background for the measurement of intra-cortical porosity. 
For each specimen, several microscope images were taken (with the number of images being dependent on the dimensions of the specimen) using an XJP300 polarizing microscope (Kozo Optics, China) with either $4 \times$ or $10 \times$ strain free objectives, and a five megapixel CMOS color camera (DCM-510, ScopeTek, China). A full wave retarder plate was used to enhance interference color generation on the unstained specimens. All images were saved in TIFF format, and, with the exception of background thresholding to change the first order red background induced by the waveplate to a black background, no post-processing or color modification was performed on any of the images.

Following image acquisition, the Image software (version 1.48 National Institutes of Health, USA) was used in order to measure areal intra-cortical porosity on the polarized light microscopic images. Total specimen surface area was measured first using the ImageJ area measuring tool, then the outline of each pore in the bone was manually traced and its area measured. Note that using either $4 \times$ or $10 \times$ microscope objectives limits the optical resolution of the microscope to approximately $3 \mu \mathrm{m}$, therefore the porosity measure did not include pores smaller than several tens of microns in diameter, as these cannot be adequately segmented at the magnifications used. The areal porosity for each acquisition was then calculated as the total area of pores divided by the total bone area (including pores).

\section{Results}

Twenty six biopsy specimens from three children were included in the study. Of these, eight had previously undergone compression testing and were assigned to Group 1, and the remaining eighteen specimens were assigned to Group 2. However, three of the Group 1 specimens failed to provide reliable mechanical compression results (two specimens were so fragile that they failed during the preconditioning cycles, and the third specimen displayed excessive surface roughness and was eliminated from the test group). Therefore five biopsy specimens were successfully tested in Group 1. Patient demographics and biopsy sites are given in Table 1. Note that all patients had undergone bisphosphonate therapy prior to specimen harvest.

Fig. 1 shows four representative polarized light images of OI bone. Note that in one of these images, the bone structure appears well aligned, but even in this image there is a region of disordered, trabecularized tissue beside the lamellar bone. Although the interference colors do not provide quantitative collagen fiber orientation in this study, the relative differences between colors in an image can be used to infer the generally high degree of disorganization in the specimens. The uneven distribution of pore sizes and shapes in the bone is evidenced by the images in Fig. 1 and also by the large variation in areal porosities measured between specimens from the same patient as shown in Table 2, which gives the mean and range of intra-cortical areal porosity measurements for all specimens from a particular biopsy site. For each of the three patients, cortical areal porosity is substantially higher than literature values for healthy cortical bone, with even the lowest porosity (Patient III) being nearly double the expected porosity value of $\sim 5 \%$. It is also interesting to note that Patient III was the only ambulant patient, and the mean porosities for the other two nonweight bearing patients are $40-50 \%$ higher again.

Table 1

OI biopsy specimen details.

\begin{tabular}{llllll}
\hline $\begin{array}{l}\text { Patient } \\
\text { ID }\end{array}$ & $\begin{array}{l}\text { Age } \\
\text { (years) }\end{array}$ & Gender & $\begin{array}{l}\text { Treatment prior } \\
\text { to surgery }\end{array}$ & Ambulant & $\begin{array}{l}\text { Biopsy location and } \\
\text { number }\end{array}$ \\
\hline I & 4.5 & M & Bisphosphonate & No & $\begin{array}{l}\text { R. femur }(\mathrm{n}=4) \\
\text { L. femur }(\mathrm{n}=8)\end{array}$ \\
II & 3 & F & $\begin{array}{l}\text { Bisphosphonate } \\
\text { L. tibia }(\mathrm{n}=7)\end{array}$ & No & R. tibia $(\mathrm{n}=7)$ \\
III & 6 & M & Bisphosphonate & Yes & \\
\hline
\end{tabular}

Fig. 2 shows a stitched reconstruction of the entire cross-section of a biopsy specimen from Patient II. This particular specimen displays large, interconnected directional pores and an overall porosity of $29 \%$, which is indicative of the highest porosities found in the sample group (refer to maximum porosity values in Table 2). Fig. 3 shows a series of fissures in the bone cross-sections. It is unclear whether these existed in vivo or are a result of biopsy removal and/or processing, therefore we refer to them as fissures rather than micro-cracks.

Figs. 4 and 5 plot the apparent level mechanical response (elastic modulus and ultimate strength respectively) versus the measured areal porosity of the specimen in question for each of the five specimens which were mechanically tested. Although the number of tested specimens is too low to permit meaningful statistical analysis of the results, these plots show a striking relationship between apparent level mechanical properties and areal porosity, with $\mathrm{R}^{2}>0.9$ in both cases. Furthermore, Fig. 6 plots apparent ultimate strength $v$ s. apparent elastic modulus for the five specimens which underwent compression testing, and again there appears to be a strong linear relationship between these two measures.

\section{Discussion}

Given the findings of previous nanoindentation studies in which both human and murine OI bone tissue have been found to exhibit similar mechanical properties to normal bone, the aim of this study was to investigate OI bone at the microstructural level using polarized light microscopy, and in particular to qualitatively assess collagen fiber organization, and to quantitatively assess areal porosity. To our knowledge, this is the first assessment of OI bone microstructure and its relation to micro-mechanical properties in human tissue.

Given the difficulties in obtaining bone biopsy from pediatric human subjects at the time of surgery, only three patients could be recruited for this study. From these three patients, we attempted to process and image all clinically harvested bone fragments where the fragments were at least a few millimeters in size. For the previously performed mechanical testing study however, only those fragments which could be reliably cut into parallelepiped blocks were tested, and so the number of mechanically tested specimens is small.

As expected, the polarized light images showed a generally high degree of collagen disorganization, with some regions in which disorganized collagen and organized collagen exist side by side in the same biopsy fragment. One limitation of the study is that the polarized light imaging used here only provided qualitative information on collagen organization, whereas it is possible to use more specialized approaches to quantitatively measure collagen alignment in bone (e.g. [18]). However, given the uncertainties in biopsy location and orientation implicit in obtaining fragments of bone removed at the time of surgery, we did not believe that the significant additional effort and resources required to obtain quantitative collagen fiber orientation were justifiable at this stage. The observations of regions of highly disorganized collagen in human OI tissue are in line with the prior histological observations [9]. Taken together with the previously mentioned nanoindentation studies by other authors [2-6], the presence of disorganized collagen alone does not seem to adversely affect tissue-level (i.e. nanoscale) mechanical integrity.

With regard to the areal cortical porosities measured in this study, the mean porosities found (between 10 and 15\%) were two to three times higher than those previously reported for healthy adult cortical bone (e.g. $0.0456 \pm 0.01$ in [19]). This phenomenon, previously named 'trabecularization' of OI bone [12], consists of a gradual loss of cortical bone's compact form leading to a structure resembling trabecular microarchitecture. In this study, trabecularization was noted in all specimens to varying extents. We note that the increased porosity found here does not appear to occur in murine OI, with a recent study finding no significant difference in porosity between OI and wild type mice [20]. Although healthy pediatric bone has been previously 

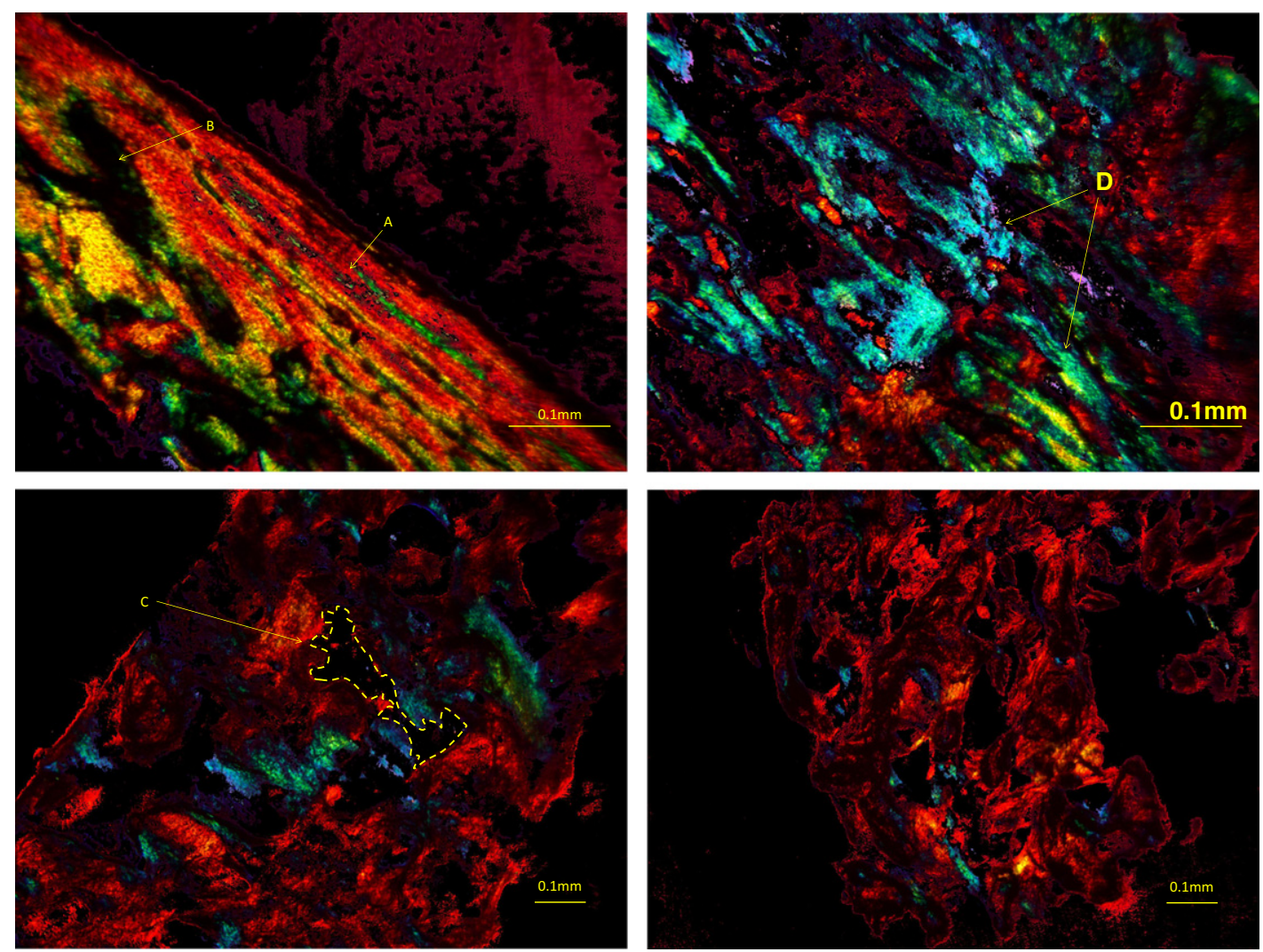

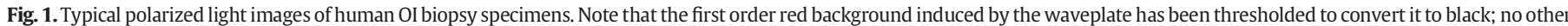

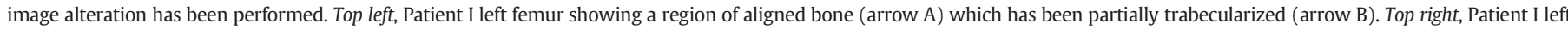

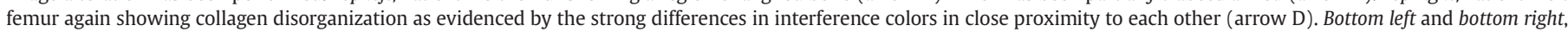

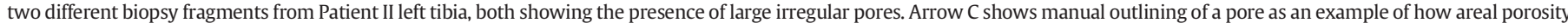
was determined.

reported to exhibit higher porosity in both cortical and trabecular bone than adult bone [21,22], the mean porosity levels in OI bone reported here appear to be higher again. Furthermore, the apparent level mechanical properties measured here are substantially lower than normal values for cortical bone [23]. Most striking is the relationship between areal porosity and mechanical properties displayed in Figs. 4 and 5. These preliminary findings on a small number of specimens point to the hypothesis that the increase in bone fragility in human OI bone is due to increased intra-cortical porosity.

Although porosity and apparent mechanical properties appear to be strongly related at the level of individual biopsy specimens, the wide range of porosity values shown in Table 2 indicates that there was a highly uneven distribution of pores between biopsy fragments from the same anatomical site. Some of this variability may be attributable to radial variations in porosity between the outer and inner cortex, although the variation in cortical porosity from approximately $9 \%$ at the outer cortex to $6 \%$ at the inner cortex reported in [21] does not fully account for the much wider range of values reported here.

An essential remark concerning the mechanical behavior of the specimens used in this study is that all patients had been treated with bisphosphonates prior to surgery. The extent to which bisphosphonate

Table 2

Areal cortical porosity for all specimens measured using polarized light microscopy.

\begin{tabular}{llllll}
\hline Patient ID & & N & Mean & \multicolumn{2}{l}{ Range (min, max) } \\
\hline I & Right & 4 & 0.145 & 0.031 & 0.292 \\
& Left & 8 & 0.160 & 0.039 & 0.271 \\
II & & 7 & 0.142 & 0.046 & 0.278 \\
III & & 7 & 0.092 & 0.034 & 0.306 \\
\hline
\end{tabular}

therapy has affected the microstructure (including the porosity) of the biopsy specimens is not known, however we note that according to [5] this treatment has no significant effect on the mechanical behavior

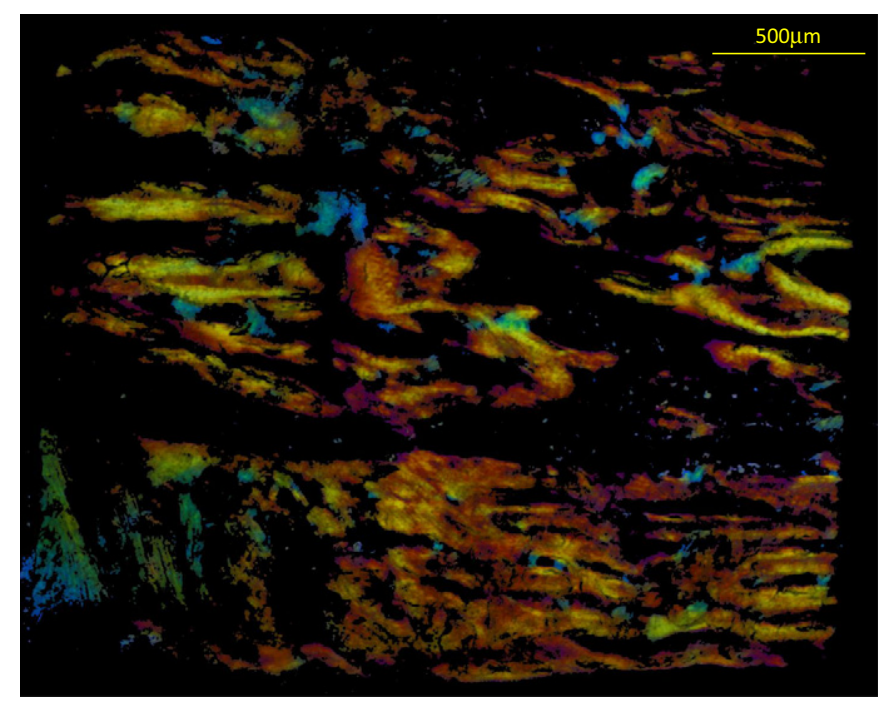

Fig. 2. Stitched polarized light image of an entire biopsy specimen from Patient II left tibia. Again the first order red background induced by the waveplate has been thresholded to convert it to black; no other image alteration has been performed. The patient was nonambulant, and this image has an areal porosity of $29 \%$; therefore it is representative of the most porous samples found in this study. Extensive trabecularization of tibial cortical bone is evident with interconnected pores and pore sizes of hundreds of microns. 

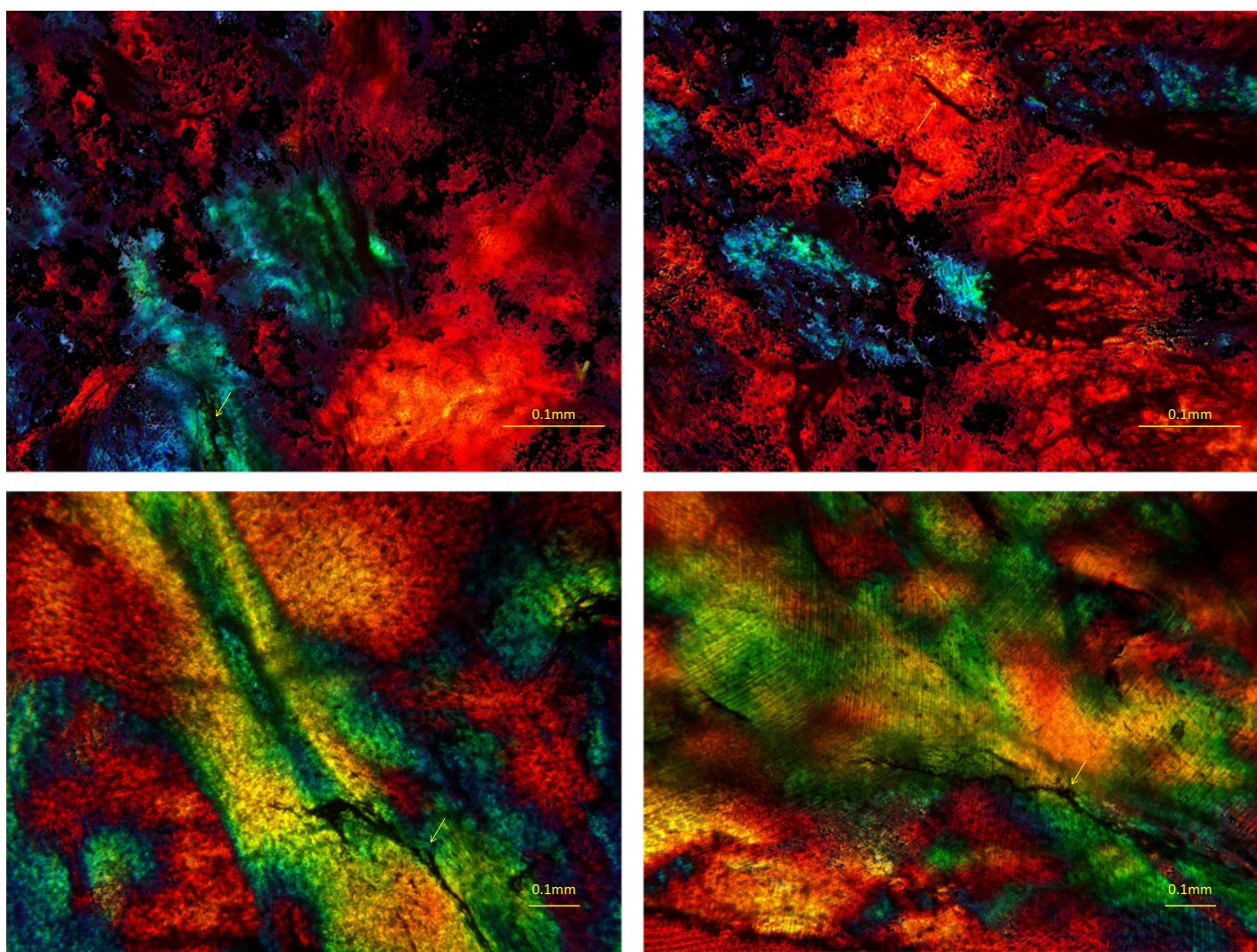

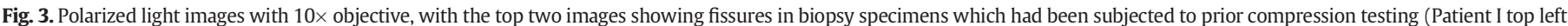

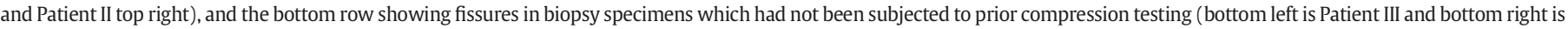

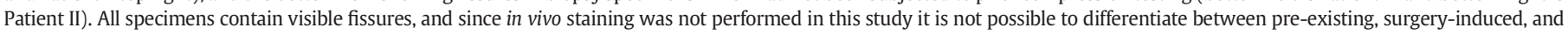
compression-induced microdamage in this study. Note some machining artifacts are visible in the bottom right image.

of the OI bone. Furthermore, since two of the patients in this study were non-ambulant, the risk of bone loss due to inactivity is high and therefore there is a possibility that the measured values for OI bone porosity were due to a combination of OI and disuse osteoporosis. This coexistence of the two diseases is reported to be common in OI patients and thus the results given here can be considered reflective of a realistic clinical situation for an OI patient [1]. As pointed out in the Results, the areal porosity for the single male ambulant patient was substantially (50\%) lower than for the two non-ambulant patients (one male and one female).

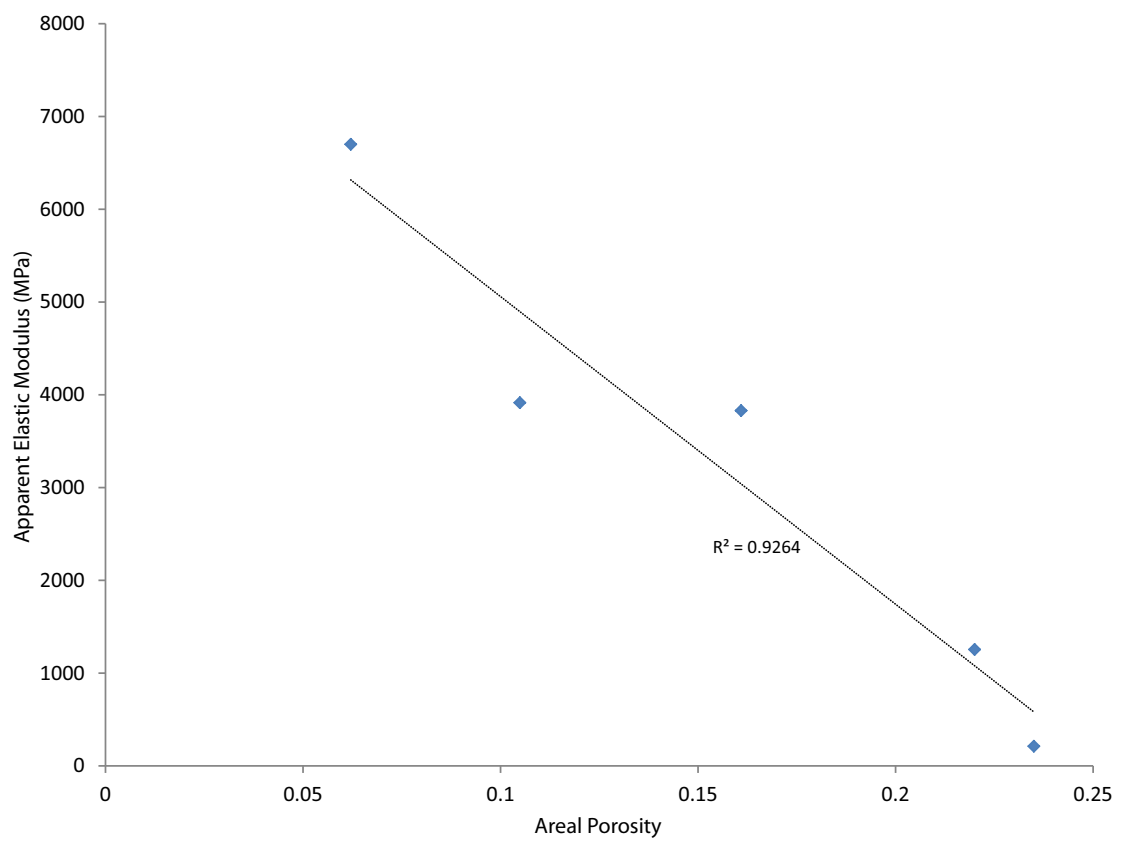

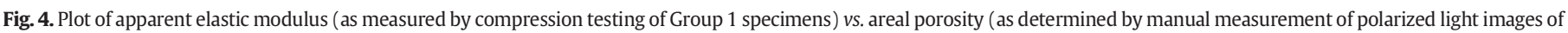
the same specimens). 


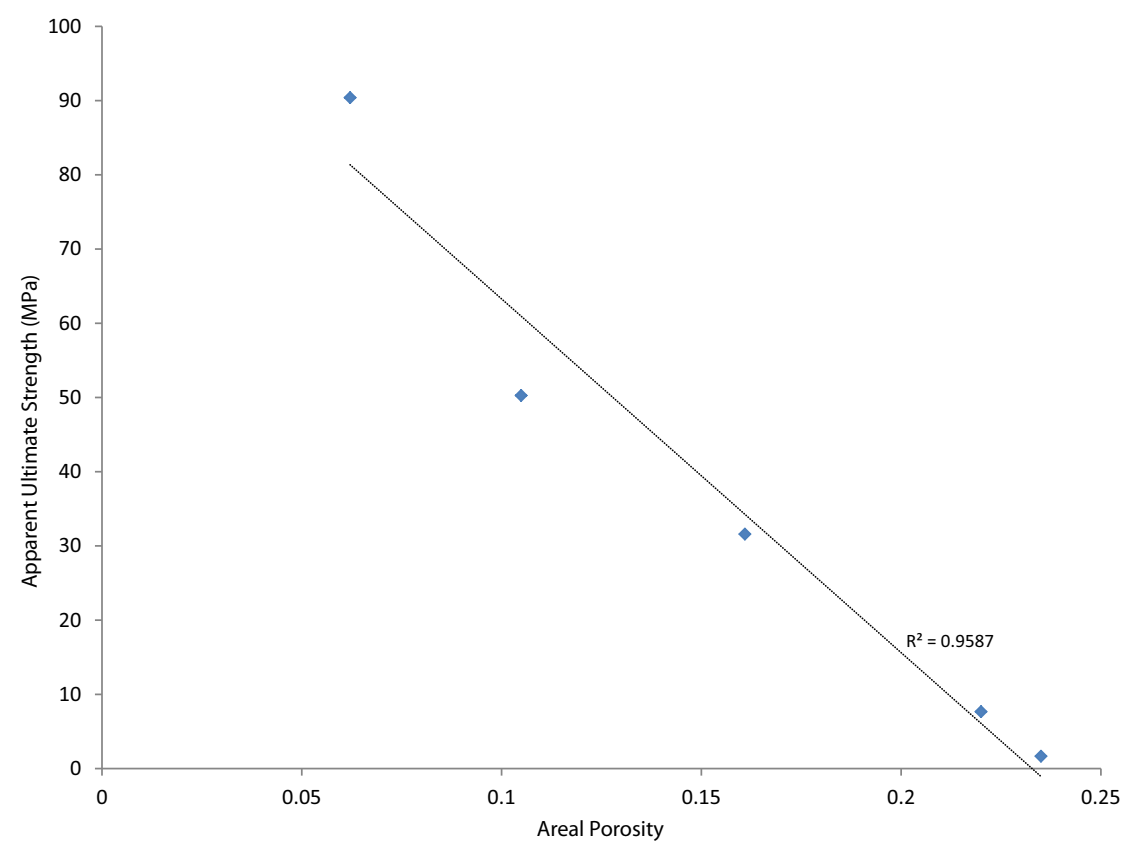

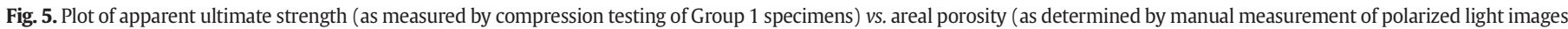
of the same specimens).

The irregular shape of the pores in the OI bone is also noteworthy. In healthy pediatric cortical bone, cortical remodeling causes the formation of large Haversian canals inside osteons [22], and Haversian porosity would be expected to be primarily responsible for the micro-porosity measured at the length scales reported here. However, the images in Figs. 1 and 2 display clear trabecularization, in which the increased porosity is not just due to the enlargement of Haversian canals, but due to the creation of new pores and fissures within the bone. These pores are often several hundred microns in size. An important implication of the large pore sizes found here is that existing high resolution in vivo imaging modalities such as PQCT may be able to adequately resolve increased micro-porosity in OI subjects. If increased intra-cortical porosity is indeed responsible for bone fragility in OI, then it may be possible to develop clinically useful subject-specific assessments of bone strength based on in vivo pQCT imaging.

There are a number of limitations to this study. Firstly, due to the nature of the resin embedding and sectioning process for polarized light microscopy, we were only able to measure porosity after mechanical testing had been performed (in the case of the Group 1 specimens). This means that some change in the microstructure of the bone could have occurred due to the compression tests, although we believe that any effect of compression on micro-porosity would be minimal due to the small $(<1 \%)$ apparent compressive strains required to reach ultimate strength. In the future it would be desirable to perform nondestructive micro-CT evaluation of the specimens prior to mechanical testing. This would also have the advantage of providing 3D porosity

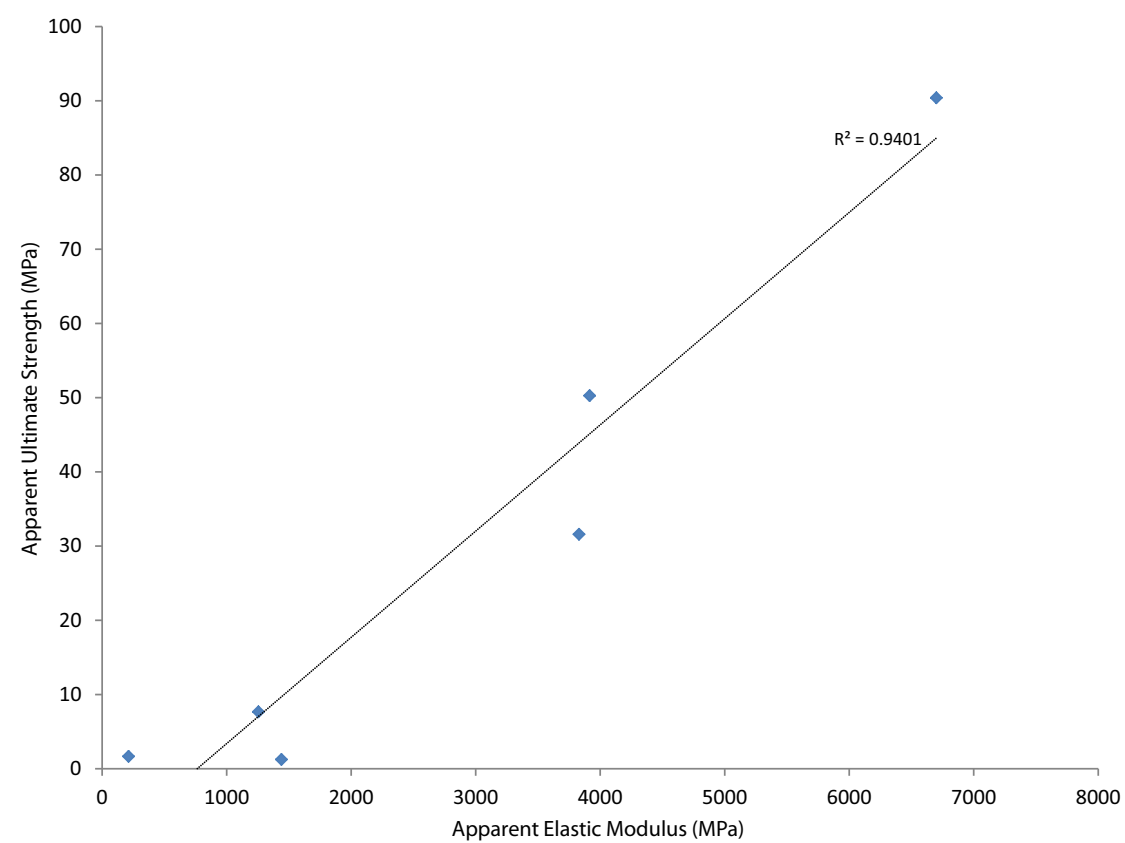

Fig. 6. Plot comparing apparent elastic modulus and apparent ultimate strength of compression tested specimens from Group 1. 
measures, although due to the small biopsy fragment thicknesses and large pore sizes in OI bone, 3D porosity measurement would require careful consideration of the concept of a representative elementary volume for assessment.

A second limitation was that no assessment of micro-damage was performed in this study. Therefore neither the effect of micro-cracks on apparent mechanical properties, nor the role which any partially open micro-cracks may have played in increasing intra-cortical porosity could be assessed. It is likely that the surgical procedure induces substantial surface micro-damage in the biopsy fragments at the time of removal, however differentiating pre-existing micro-damage from that induced by surgical removal and specimen preparation would have required in vivo labeling which was beyond the scope of this study.

A third limitation is that due to the nature of biopsy specimen collection during surgery, the orientation and location of the retrieved bone fragments relative to the orientation of the patient's femur or tibia was not well known. As mentioned in the Materials and methods, it was generally possible to visually estimate the bone fabric direction prior to cutting the parallelepipeds for compression testing, however detailed orientation and location information for each small fragment (e.g. inner vs. outer cortex) was not obtainable. Given the high local variability in OI bone porosity and the frequent appearance of directional pores (Fig. 2), improved orientation information would be valuable in future studies.

In conclusion, the polarized light microscopy performed in this study indicates that pediatric OI bone exhibits extensive collagen disorganization and trabecularization, with increased porosity and reduced mechanical integrity compared to healthy bone. Importantly, micromechanical properties tentatively appear to be driven by increased intra-cortical porosity. Large pores in OI bone highlight the possibility of in vivo PQCT imaging for patient-specific bone fragility assessment in the future. We hope that the initial results given here will lead to further investigation of the role of micro-porosity in OI bone fragility by research groups with clinical access to human OI bone tissue.

\section{References}

[1] Glorieux FH. Osteogenesis imperfecta. Best Pract Res Clin Rheumatol 2008;22: 85-100.

[2] Albert C, Jameson J, Toth JM, Smith P, Harris G. Bone properties by nanoindentation in mild and severe osteogenesis imperfecta. Clin Biomech 2012:28:110-6.
[3] Fan Z, Smith PA, Eckstein EC, Harris GF. Mechanical properties of OI type III bone tissue measured by nanoindentation. J Biomed Mater Res 2006;A 79:71-7.

[4] Fan Z, Smith PH, Harris GF, Rauch F, Bajorunaite R. Comparison of nanoindentation measurements between osteogenesis imperfecta type III and type IV and between different anatomic locations (femur/tibia versus iliac crest). Connect Tissue Res 2007;48:70-5

[5] Weber M, Roschger P, Fratzl-Zelman N, Schöberl T, Rauch F, Glorieux FH, et al. Pamidronate does not adversely affect bone intrinsic materials properties in children with osteogenesis imperfecta. Bone 2006;39:616-22

[6] Imbert L, Aurégan JC, Pernelle K, Hoc T. Mechanical and mineral properties of osteogenesis imperfecta human bones at the tissue level. Bone 2014;65:18-24.

[7] Nyman JS, Reyes M, Wang X. Effect of ultrastructural changes on the toughness of bone. Micron 2005;36:566-82.

[8] Davis MS, Kovacic BL, Marini JC, Shih AJ, Kozloff KM. Increased susceptibility to microdamage in Brtl/+ mouse model for osteogenesis imperfecta. Bone 2012;50: 784-91.

[9] Rauch F, Travers R, Parfitt AM, Glorieux FH. Static and dynamic bone histomorphometry in children with osteogenesis imperfecta. Bone 2000:26:581-9.

[10] Marotti G. A new theory of bone lamellation. Calcif Tissue Int 1993;53(Suppl. 1): S47-56.

[11] Dong XN, Zoghi M, Van Q, Wang X. Collagen mutation causes changes of the microdamage morphology in bone of an OI mouse model. Bone 2010;47:1071-5.

[12] Jones SJ, Glorieux F, Travers R, Boyde A. The microscopic structure of bone in normal children and patients with osteogenesis imperfecta: a survey using backscattered electron microscopy. Calcif Tissue Int 1999;64:8-17.

[13] G. Antherieu. Mechanical characterization of child cortical bone for the study of osteogenesis imperfecta, Unpublished Masters Thesis, Laboratoire de Biomecanique, Arts et Metier ParisTech (ENSAM), Paris, France, 2012.

[14] Goulam Houssen Y, Gusachenko I, Schanne-Klein M-C, Allain J-M. Monitoring micrometer-scale collagen organization in rat-tail tendon upon mechanical strain using second harmonic microscopy. J Biomech 2011;44:2047-52.

[15] An YH, Martin KL. Handbook of histology methods for bone and cartilage. Totowa, New Jersey: Humana Press; 2003.

[16] Ellis AE. Corrected formulation for Spurr low viscosity embedding medium using the replacement epoxide ERL 4221. Microsc Microanal 2006;12(Suppl. 2).

[17] Bromage TG, Goldman HM, McFarlin SC, Warshaw J, Boyde A, Rigos CM. Circularly polarized light standards for investigations of collagen fiber orientation in bone. Anat Rec B 2003;274:157-68.

[18] Spiesz EM, Kaminsky W, Zysset PZ. A quantitative collagen fibers orientation assessment using birefringence measurements: calibration and application to human osteons. J Struct Biol 2011;176:302-6.

[19] Feik SA, Thomas CDL, Clement JG. Age-related changes in cortical porosity of the midshaft of the human femur. J Anat 1997;191:407-16.

[20] Carriero A, Doube M, Vogt M, Busse B, Zustin J, Levchuk A, et al. Altered lacunar and vascular porosity in osteogenesis imperfecta mouse bone as revealed by synchrotron tomography contributes to bone fragility. Bone 2014;61:116-24.

[21] Schnitzler CM, Mesquita JM, Pettifor JM. Cortical bone development in black and white south African children: iliac crest histomorphometry. Bone 2009;44:603-11.

[22] Rauch F, Travers R, Glorieux FH. Intracortical remodeling during human bone development - a histomorphometric study. Bone 2007:40:274-80.

[23] Ohman C, Baleani M, Pani C, Taddei F, Alberghini M, Viceconti M, et al. Compressive behaviour of child and adult cortical bone. Bone 2011;49:769-76. 